\title{
Data Exploitation Model in a Health Ecosystem to Support Formal and Informal Caregivers ${ }^{+}$
}

\author{
Samuel Marcos-Pablos *, Alicia García-Holgado and Francisco José García-Peñalvo \\ GRIAL Research Group, Computer Science Department, University of Salamanca, 37008 Salamanca, Spain; \\ aliciagh@usal.es (A.G.-H.); fgarcia@usal.es (F.J.G.-P.) \\ * Correspondence: samuelmp@usal.es; Tel.: +34-923294500 (ext. 3433) \\ + Presented at the 13th International Conference on Ubiquitous Computing and Ambient Intelligence \\ UCAmI 2019, Toledo, Spain, 2-5 December 2019.
}

Published: 20 November 2019

\begin{abstract}
Business interactions are rarely taken into account in the early stages of development of software ecosystems in the health sector, which causes these kinds of solutions to stay as conceptual proposals or be rapidly discontinued after deployment. In the present paper, we describe our approach towards the exploitation of a technological ecosystem focused on learning and knowledge management processes to develop and enhance the caregiving competences of formal and informal caregivers, both at home and in care environments. The definition of the business process has been done using the Business Process Model and Notation (BPMN) standard, which allows to further include the business model in the definition of the data taxonomy.
\end{abstract}

Keywords: health ecosystem; metamodel; caregivers; business data; business process; BPMN

\section{Introduction}

Nowadays, it is a fact that Europe is an ageing society. While world population size is expected to increase to 10 billion people at the end of the 21st century, in recent decades the population has declined in some regions of the world [1]. In this context, informal caregivers play an important role. They prevent the institutionalization of the dependent persons, enabling them to stay at home and thus reducing care costs. However, this role has a high health impact on informal caregivers, linked to suffering severe health and mental health problems, such as overload, depression, or anxiety, significantly reducing their quality of life and increasing social isolation and family stress.

Psychoeducation is an alternative solution to this social problem [2]; combining information and behavior management training with attention to family support can have significant positive effects for caregivers. However, sometimes it is hard to access to these interventions, not only for geographical reasons but also for the socioeconomic situation of informal caregivers and their families. Information and Communication Technologies (ICT) have the potential to improve accessibility by supporting virtual coaching and the delivery of psychoeducation interventions [3]. In this context, the current paper presents a technological ecosystem focused on learning and knowledge management processes to develop and enhance the caregiving competences of formal and informal caregivers, both at home and in care environments.

During the last decade, technological ecosystems [4] have emerged as a technological solution based on a set of software components that interact in some way to give rise to more complex systems. In fact, the European Union has funded several projects to support formal and informal caregivers in different ways [5,6], but none is focused on supporting informal caregivers through psychoeducation. Moreover, proposed or developed health ecosystems tend to have problems when it comes to being deployed in the real world. This means that they are usually discontinued despite their great potential. For example, a recent study [7], which analyzed 37 papers focused on platforms 
providing care and assistance services under a technological ecosystems' perspective, showed that just three papers $(8 \%)$ described an ecosystem that was deployed and tested with real users under real world conditions (out of the lab). This may be because the vast majority of such proposals do not take into account the concept of value co-creation within the ecosystem, and lack a business perspective when defining the relevant data to be exploited. They are usually focused on providing services for the patient end user, but forget the rest of the stakeholders when it comes to ecosystem exploitation.

To overcome these limitations, the present work focuses on providing an approach towards the exploitation of the proposed ecosystem based on the modeling of the business process. The process starts from a general business model based on the Osterwalder and Pigneur model [8], after which a description of the stakeholders and associated business processes are drawn up. The definition of the business process is done using the Business Process Model Notation (BPMN) standard. The use of this standard allows to include the business model in the definition of the data taxonomy, which may be then employed as a starting data model in the software development process [9].

The rest of the paper is organized as follows. Section two describes the health ecosystem for caregivers. The third section analyzes the considered stakeholders in the business model. Sections four and five describe the business process for primary, secondary and tertiary stakeholders along with the associated data susceptible for exploitation. The last section summarizes the main conclusions of this work.

\section{Health Ecosystem for Caregivers}

The technological ecosystem consists of three main structures: software, organizational and business. The health ecosystem's software structure is composed of a set of software components and a set of input streams that represents the human factor as part of the technological ecosystem (see Figure 1).

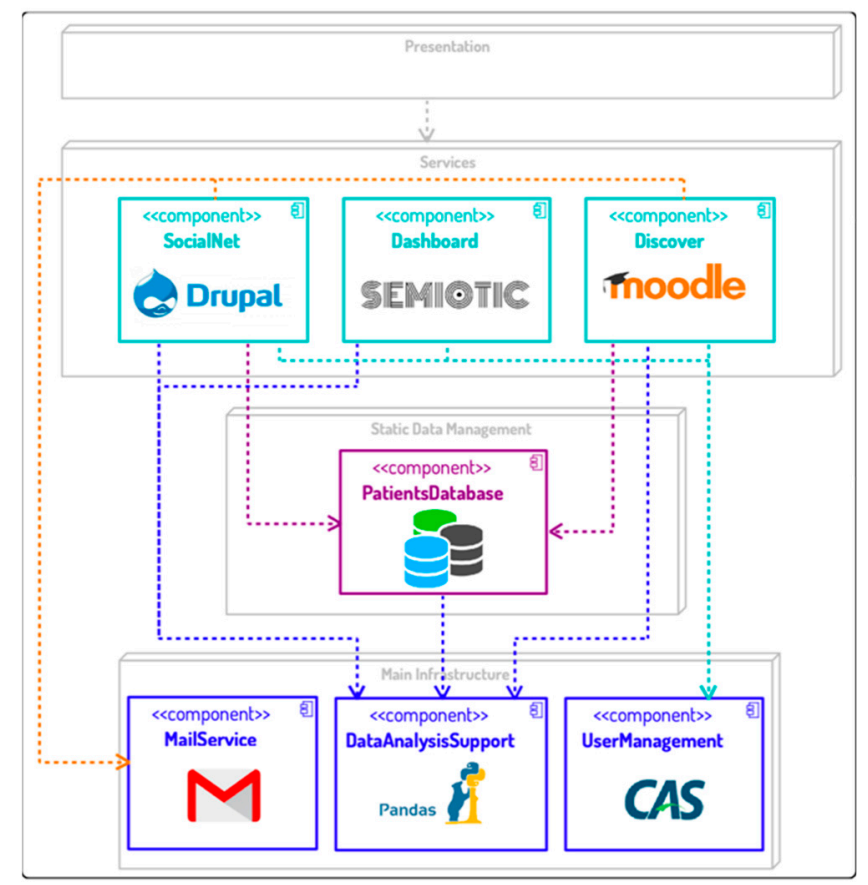

Figure 1. Architecture of the health ecosystem for caregivers.

The architecture is based on the proposal validated in previous works [10]. The software components are organized into four layers. The first layer is focused on the presentation through a unique interface, and for a consistent design and enhanced user experience (UX). The second layer, the services layer, is focused on the software components that offer a set of services to the final users of the ecosystem: an online tool that provides a private social network for patients private areas 
(called walls), which are managed by (in)formal caregivers and care managers; a learning platform based on Moodle to support psychoeducation processes, including knowledge needs of the informal caregivers, specialized training for formal caregivers and access to a community of equals and experts that can help them [11]; a dashboard to provide tools based on visual analysis to support decision-making processes. The third layer provides tools to centralize information needed by other components of the ecosystem. It includes a database that stores data about the patients: treatments, caregivers visits, location etc. Finally, the infrastructure layer provides a set of services that are used by the software components from other layers: mail service, user management system to centralize the user access and information and data analysis component to process the data from other tools and generate the datasets that are an input for the dashboard. Moreover, the data analysis module has a strong component focused on Learning Analytics.

Regarding the organizational structure, the definition of the business structure provides the base of the input streams included in the architecture, thus, it is directly linked to the business structure described in the following sections. The business structure model is based on the approach developed by Osterwalder and Pigneur [8] and is described in detail in [11]. It consists in the fulfillment of a Business Model Canvas (BMC) that describes the interrelation of nine components of a business model. The proposed BMC is based in a literature review aimed to identify how technological ecosystem proposals address the business component [7], along with a systematic mapping study of the most relevant EU funded projects related to the health domain. The outcomes of these reviews (trends, lacks and opportunities, involved actors etc.) were applied to define the different components of the BMC.

\section{The Stakeholder Cloud}

One of the main distinctions of technological ecosystems when compared to other types of business models resides in the strong involvement of the different ecosystem actors in value cocreation. In this sense, the first step to identify the ecosystem's relevant data subject to exploitation is the correct classification of stakeholders. Stakeholders' clustering into different groups and their nomenclature has been revisited over the years [12]. In the province of AAL (Ambien Assisted Living), three fundamental types of stakeholders are distinguished [13]: (a) Primary end-users: person who is using a product or health service; (b) Secondary users: people or organizations that that are accessing or using the ecosystem solutions for the benefit of primary end-users; (c) Tertiary final users: institutions and private or public organizations that are not directly in contact with the AAL solution, but that in some way contribute and benefit from their organization, budging or assessment (e.g., organizers of public sector services, social security systems, insurance companies etc.).

Given the nature of our ecosystem, the classification approach commonly used by the authors is the AAL classification [13]. However, in terms of data extraction and exploitation, it is necessary to also take into account the business approach (more focused on business activities). Additionally, given the software component of the platform, we should take into account the software point of view.

As shown in Figure 2, the considered groups of stakeholders differ from the proposed AAL classification. We review the AAL definition of primary end-user, because in our case, the ecosystem is mainly focused on caregivers. In addition, given that caregiver organizations incorporate formal caregivers and their managers coordinators, this type of organization is partly considered to be within the group of secondary actors. Finally, the figure of platform orchestrator must include the actors responsible for the ecosystem governance from the software point of view. The taken approach to identify the exploitable data is to search within the business processes those data which generate added value for each group of stakeholders. 


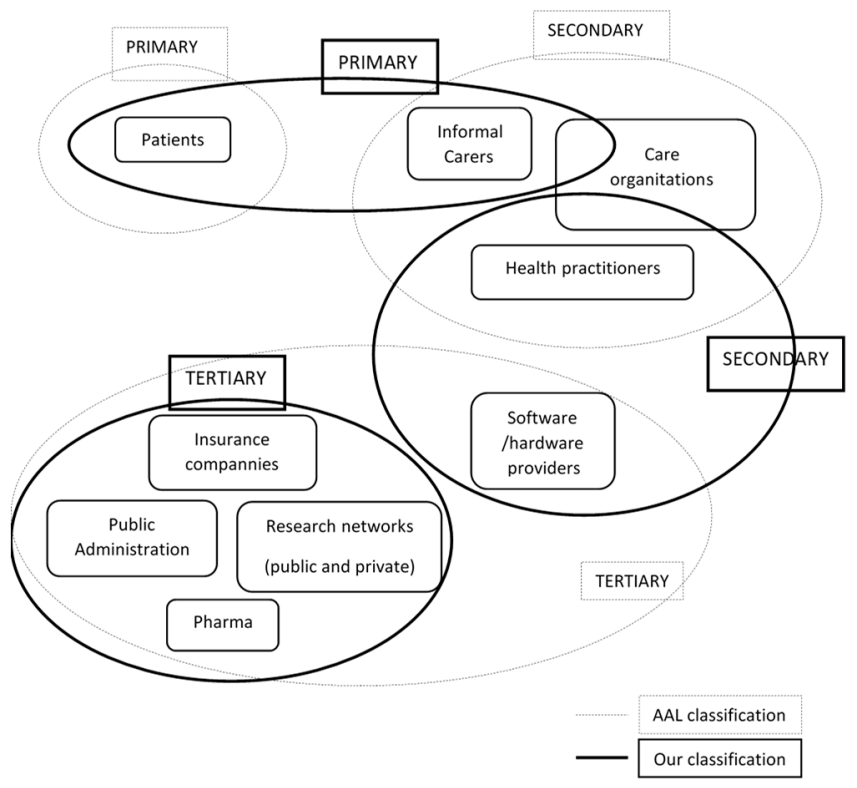

Figure 2. The stakeholders' cloud: global picture of the stakeholders in our ecosystem.

\section{Primary Stakeholders' Approach to Exploitable Data}

Taking into account the stakeholders' cloud, our primary users are mainly (in)formal caregivers and patients. The business process for primary stakeholders is based on the combined use of the social network of patients, relatives and caregivers (SocialNet) and the learning platform (Discover). To describe the business process model, we used a BPMN diagram (Figure 3). It includes three lanes in order to show the collaborative process between the primary users, the data management system and the caregiver managers.

For primary users (upper pool) the process starts with the onboarding of new users. In this step, a user's profile data is requested (age, health status, geographical situation, previous caregiving knowledge etc.); if the user accesses the training platform, an initial training itinerary is recommended for the user based on these data. Based on the continued use of the Discover and SocialNet tools, the training itinerary is iteratively improved and adapted to the user training needs. In this stage, the caregiver can choose between two paths:

- Unassisted support: using tools that allow the user to follow his own learning path without mentoring: evaluation questionnaires, personalized searches and content recommendation.

- Assisted Support: either provided by experts in the mentoring program that support the caregivers in scheduled sessions or through the social network, or through a Smart assistant that is nourished from the ecosystem interactions answers questions 24/7.

The data extracted from the evaluation questionnaires, personalized queries, content recommendation, and mentoring activities serve as input to a database that stores real time data. Then, a clustering module based on Learning Analytics [14] and a dashboard is launched periodically. This module provides information based on all combined inputs (detected problems, suggested themes of interest, the analysis of the sessions with the mentors, etc.) in order to improve the learning itinerary. The combination of the AI-based and dashboard-based Learning Analytics provides feedback and learning content indications to other ecosystem tools: recommendations of follow-up training contents depending on the preference/utility for a particular user, advanced and customized searches, improved Smart Assistant and relevant data that provides additional added value for secondary and tertiary actors (which will be covered on next section).

As mentioned in the previous section, the care organizations are considered to be among the primary and secondary stakeholders, since they include both the formal caregivers who are part of said organizations (primary), and the managers of said caregivers (secondary). The figure of the care managers in this model (bottom lane) comprises both the organization managers as well as 
specialized personnel capable of supporting the learning process. Thus, the BPMN shows how care managers can interact with the system by providing support to primary users while at the same time they can obtain relevant information through the dashboard. Additionally, the visualized dashboard information can be combined so that their feedback can be adapted to the specific needs of the caregiver and patient. The visualized data can also help them to make better management decisions (scheduling, reassignment of patients etc.). Finally, every time the services of the social network, dashboard or learning platform are accessed, the system sends personalized data to the user interface (e.g., next suggested learning actions, personalized searches, access to stored data).

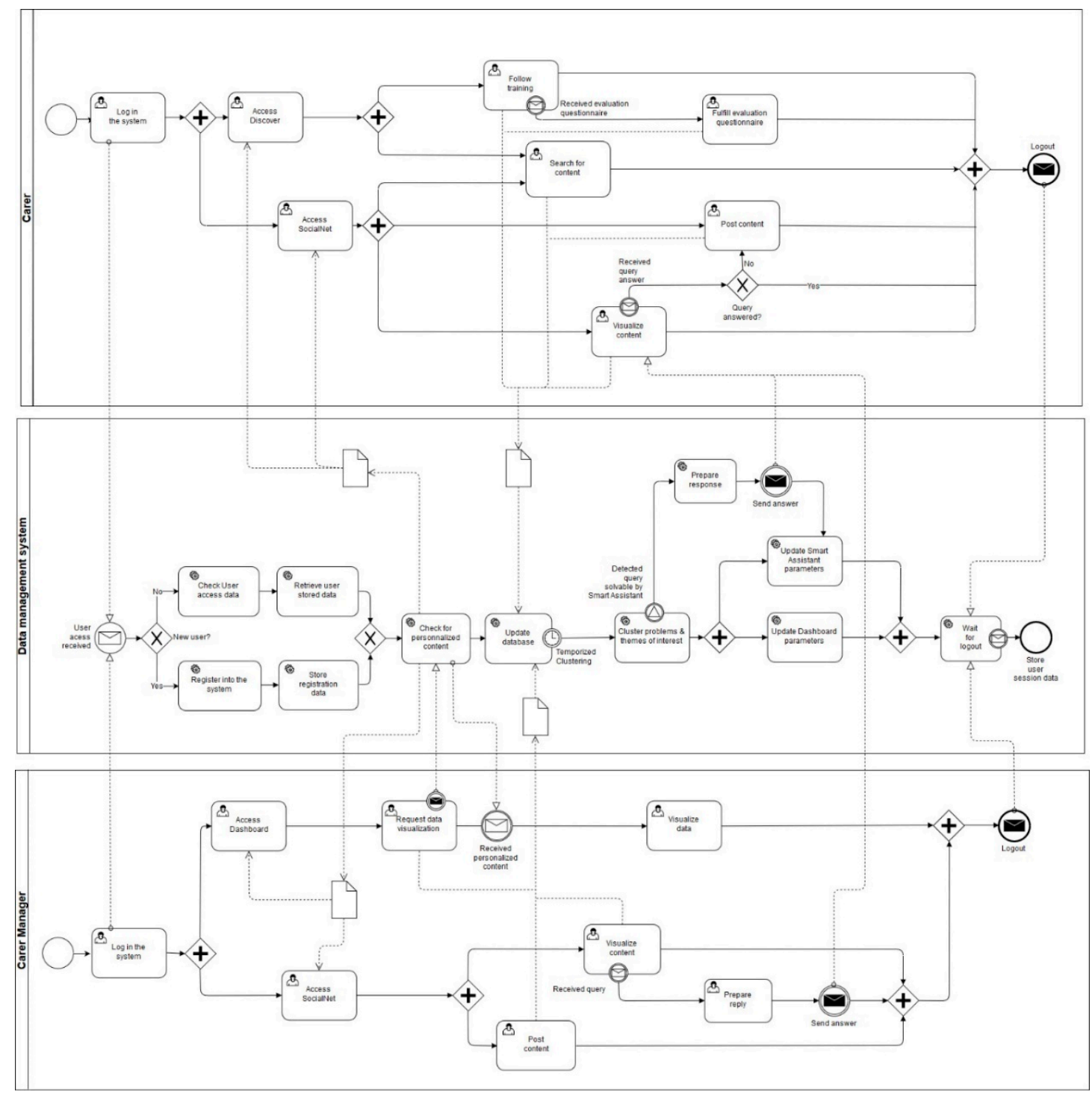

Figure 3. Business Process Model and Notation (BPMN) model for primary stakeholders (caregivers) and care managers.

\section{Secondary and Tertiary Stakeholders' Approach to Exploitable Data}

From a global perspective, for secondary and tertiary users, the strength of the proposed ecosystem lies in the ability to simultaneously combine data of different nature (e.g., ethnographical, clinical, medical) [15]. In the current ecosystem approach, exploitable data refer not only to data subject to commercialization, but also to data that may be of interest and generate added value to stakeholders. In that sense, the figure of "expert" patient/caregiver (non-expert person but with an increased access to Information and Communication Technologies (ICTs) solutions to enhance their own treatment) is foreseen to have a great impact in future provision of medical care. 
Thus, in order to exploit the data within the ecosystem, it is necessary to match the activities of the primary users with the value sought by the secondary and tertiary users and vice versa. With that goal in mind, data directly collected from the primary users must be considered on the one hand, such as: patient clinical/medical data, treatment adherence and disease evolution, patients and caregivers' ethnographical data, etc. On the other hand, from the interaction of the primary users with the ecosystem services, the secondary and tertiary users can also use inferred data. These data can be extracted by combining different sources of information, such as the questions raised by users in the forums, experiences and shared use cases, the contents sought, the training evolution of the caregiver, etc.

More specifically, the data that may be of interest for each of the different identified stakeholders include:

- Health practitioners: their main objective is to improve decision-making by identifying better treatments and discard the least efficient ones. They will seek to detect adherence to treatment, its effectiveness and finally to improve the quality of life of primary users.

- Research Networks: their objective is to acquire new knowledge searching for new solutions to unresolved medical problems investigating the interaction of different factors within the data in order to understand, for example, how they influence the risk for disease or disease expression [15].

- Care managers: as secondary users will also seek to reduce costs and resource expenses. To do so, they will seek to acquire data that helps them to improve learning contents, give better support in the training process and consequently improve the mentoring programs.

- Public administration and insurance companies: they will seek for a better understanding of the health economic impact by disease category and patient sub-type, which would provide additional incentives to invest in treatment and research and to look for preventive treatments.

- Pharma: in terms of drug treatment, some diseases as is the case of dementia are only susceptible of symptomatic treatment. In these cases, big data can provide a better understanding of how to best use the available therapies.

- Software and hardware providers: include both service providers and the healthcare technology sector. Service providers will be interested in obtaining more relevant data on the use of the platform for monitoring system performance (time slots of maximum platform usage, technologies employed for accessing the system etc.). From the point of view of developers of medical hardware and software solutions (e.g., orthopedics, biomedical measuring systems etc.), data on the evolution of patients as a result of the use of their devices can be obtained from the social network.

- Other: given the evolutive nature of the ecosystem, the ability to extend its functionality and include new services, as well as the existence of the social network can lead to new business opportunities (e.g., specific advertising content for business focused on the elderly, such as travel agencies, etc.).

Taking the above into account, Figure 4 shows the general business model for secondary and tertiary stakeholders condensed into a single BPMN diagram as the business process is similar for all of them. Differences may be found in the restrictions for the different stakeholders that arise from the data governance during the data lifecycle (e.g., data protection regulations, ethics).

As can be seen in the BPMN, in order the stakeholders to access the data, a first stage is required during which the system determines the recommended contents and the accessible data to be visualized on the dashboard (based on the data exchanged with the system by the particular user). Once the desired content has been visualized, the user can export the data for later use in other systems (for example, dedicated databases belonging to research networks). Both the request for display and export data are system services and therefore are modeled by an exchange of messages between the user and the data management system. 

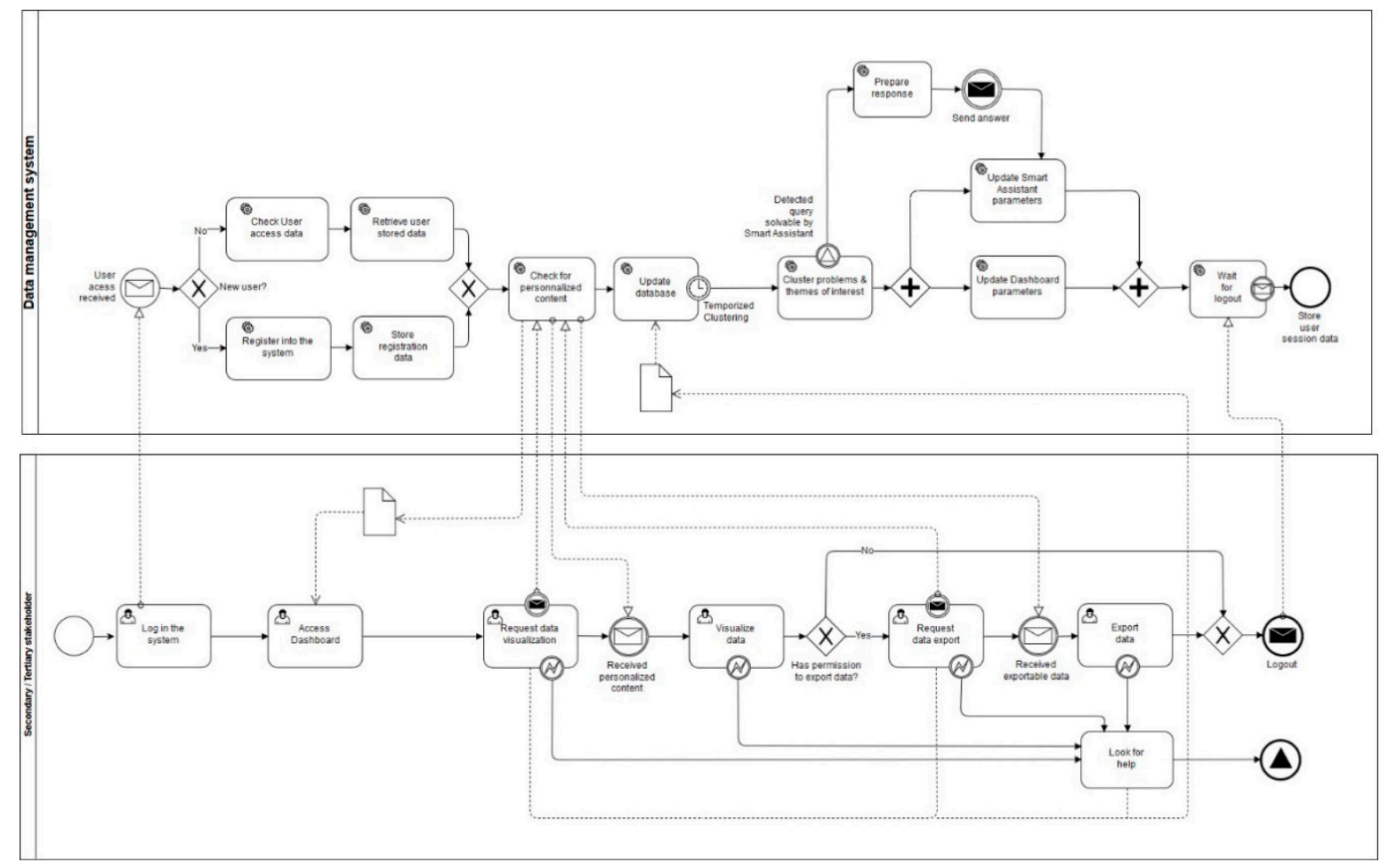

Figure 4. General BPMN model for secondary and tertiary stakeholders.

\section{Conclusions and Future Work}

The current trend in Information technology (IT) solution providers (encompassing more than just the health sector) is to move from fragmented services to progressively more integrated services, which are likely to be provided by multiple stakeholders through well-elaborated collaboration mechanisms. As such, the field of technological ecosystems has emerged as the study of the complex interaction between technological devices (ambient sensors, wearables, medical devices, etc.) and extensible technological frameworks and architectures on the one hand, and organizations, users, customers, developers and businesses on the other.

However, these business interactions are scarcely taken into account at the early stages of development of software ecosystems in the health sector, which causes these kinds of solutions to stay as conceptual proposals or be rapidly discontinued after deployment. In the present paper, we have provided an approach towards the exploitation of a technological ecosystem focused on learning and knowledge management processes to develop and enhance the caregiving competences of formal and informal caregivers, both at home and in care environments. The definition of the business process has been done using the BPMN standard, which allows to include the business model in the definition of the data taxonomy, which may then be employed as a starting data model in the software development process.

Future lines will include the formalization of the data taxonomy. Given the interoperable and scalable nature of the platform, together with the differences between the health and care provision policies that may vary between geographical regions, obtaining and processing data becomes a more complex task than in conventional software platforms. Therefore, since medical data are combined with data on the use of the platform, the actions to be taken include: determining the best practices to obtain data within the ecosystem data flow; establishing the data that could be commercialized in each region, taking into account the privacy rules and ethics applicable in each case; developing methods and tools for the automated but anonymized (or pseudo-anonymized) storage and analysis of the data during the business process. 
Author Contributions: Conceptualization, S.M.-P., A.G.-H. and F.J.G.-P.; Methodology, S.M.-P. and A.G.-H.; Writing-Original Draft Preparation, S.M.-P. and A.G.-H.; Writing-Review and Editing, F.J.G.-P.; Supervision, F.J.G.-P.; Project Administration, F.J.G.-P.; Funding Acquisition, F.J.G.-P.

Funding: This research was partially funded by the Spanish Government Ministry of Economy and Competitiveness throughout the DEFINES project grant number [TIN2016-80172-R] and the Ministry of Education of the Junta de Castilla y León (Spain) throughout the T-CUIDA project [SA061P17].

Acknowledgments: The authors would like to thank the InterAction and eLearning Research Group (GRIAL) for its support to conduct the present research https://grial.usal.es.

Conflicts of Interest: The authors declare no conflict of interest.

\section{References}

1. Bongaarts, J. Human population growth and the demographic transition. Philos. Trans. R. Soc. B Biol. Sci. 2009, 364, 2985-2990, doi:10.1098/rstb.2009.0137.

2. Ostwald, S.K.; Hepburn, K.W.; Caron, W.; Burns, T.; Mantell, R. Reducing Caregiver Burden: A Randomized Psychoeducational Intervention for Caregivers of Persons with Dementia. Gerontologist 1999, 39, 299-309, doi:10.1093/geront/39.3.299.

3. Pot, A.M.; Blom, M.M.; Willemse, B.M. Acceptability of a guided self-help Internet intervention for family caregivers: Mastery over dementia. Int. Psychogeriatr. 2015, 27, 1343-1354, doi:10.1017/S1041610215000034.

4. Jansen, S.; Finkelstein, A.; Brinkkemper, S. A Sense of Community: A Research Agenda for Software Ecosystems. In Proceedings of the 31st International Conference on Software Engineering-Companion Volume (ICSE-Companion 2009), Vancouver, BC, USA, 16-24 May 2009; pp. 187-190, doi:10.1109/ICSE-COMPANION.2009.5070978.

5. García-Holgado, A.; Marcos-Pablos, S.; Therón, R.; García-Peñalvo, F.J. Technological ecosystems in the health sector: A mapping study of European research projects. J. Med. Syst. 2019, 43, doi:10.1007/s10916-019-1241-5.

6. Marcos-Pablos, S.; García-Holgado, A.; García-Peñalvo, F.J. Trends in European research projects focused on technological ecosystems in the health sector. In Proceedings of the 6th International Conference on Technological Ecosystems for Enhancing Multiculturality (TEEM 2018), Salamanca, Spain, 24-26 October 2018; García-Peñalvo, F.J., Ed.; ACM: New York, NY, USA, 2018; doi:10.1145/3284179.3284263.

7. Marcos-Pablos, S.; García-Peñalvo, F.J. Technological Ecosystems in Care and Assistance: A Systematic Literature Review. Sensors 2019, 19, 708, doi:10.3390/s19030708.

8. Osterwalder, A.; Pigneur, Y. Business Model Generation: A Handbook for Visionaries, Game Changers, and Challengers; Wiley: Hoboken, NJ, USA, 2010.

9. Leshob, A. Towards a business-pattern approach for UML models derivation from business process models. In Proceedings of the 13th IEEE International Conference on e-Business Engineering (ICEBE 2016), Macau, China, 4-6 November 2016; Guo, J., Cai, H., Fei, X., Chao, K., Chung, J., Eds.; IEEE Computer Society: Washington, DC, USA, 2016; pp. 244-249, doi:10.1109/ICEBE.2016.049.

10. García-Holgado, A.; García-Peñalvo, F.J. Architectural pattern to improve the definition and implementation of eLearning ecosystems. Sci. Comput. Program. 2016, 129, 20-34, doi:10.1016/j.scico.2016.03.010.

11. García-Holgado, A.; Marcos-Pablos, S.; García-Peñalvo, F.J. A Model to Define an eHealth Technological Ecosystem for Caregivers. In New Knowledge in Information Systems and Technologies; WorldCIST'19 2019. Advances in Intelligent Systems and Computing; Rocha, Á., Adeli, H., Reis, L., Costanzo, S., Eds.; Springer: Cham, Switzerland, 2019; Volume 932, pp. 422-432.

12. Sirgy, M.J. Measuring Corporate Performance by Building on the Stakeholders Model of Business Ethics. J. Bus. Ethics 2002, 35, 143-162.

13. Nedopil, C.; Schauber, C.; Glend, S. AAL Stakeholders and their requirements Ambient Assisted Living Association Knowledge Base, 2013. Available online: http://www.aal-europe.eu/wp-content/uploads/2015/ 02/AALA_Knowledge-Base_YOUSE_online.pdf (accessed on 11 April 2019). 
14. Fidalgo-Blanco, Á.; Sein-Echaluce, M.L.; García-Peñalvo, F.J.; Conde, M.Á. Using Learning Analytics to improve teamwork assessment. Comput. Hum. Behav. 2015, 47, 149-156, doi:0.1016/j.chb.2014.11.050.

15. Anderson, G.; Buckle, R.; Favrin, G.; Friend, S.; Geschwind, D.; Hu, H.; Oliver, S.; Petersen, R.; Rossor, M.; St George-Hyslop, P.; et al. Dementia Research and Care: Can Big Data Help? OECD Publishing: Paris, France, 2015, doi:10.1787/9789264228429-6-en.

(C) 2019 by the authors. Licensee MDPI, Basel, Switzerland. This article is an open access article distributed under the terms and conditions of the Creative Commons Attribution (CC BY) license (http://creativecommons.org/licenses/by/4.0/). 